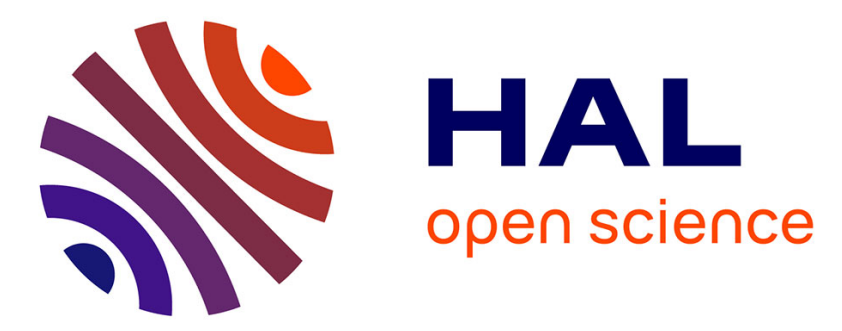

\title{
BMP4 patterns Smad activity and generates stereotyped cell fate organisation in spinal organoids
}

\author{
Nathalie Duval, Célia Vaslin, Tiago Barata, Youcef Frarma, Vincent \\ Contremoulins, Xavier Baudin, Stéphane Nédélec, Vanessa Ribes
}

\section{- To cite this version:}

Nathalie Duval, Célia Vaslin, Tiago Barata, Youcef Frarma, Vincent Contremoulins, et al.. BMP4 patterns Smad activity and generates stereotyped cell fate organisation in spinal organoids. Development (Cambridge, England), 2019, 146 (14), pp.dev175430. 10.1242/dev.175430 . pasteur-03254035

\section{HAL Id: pasteur-03254035}

\section{https://hal-pasteur.archives-ouvertes.fr/pasteur-03254035}

Submitted on 8 Jun 2021

HAL is a multi-disciplinary open access archive for the deposit and dissemination of scientific research documents, whether they are published or not. The documents may come from teaching and research institutions in France or abroad, or from public or private research centers.
L'archive ouverte pluridisciplinaire HAL, est destinée au dépôt et à la diffusion de documents scientifiques de niveau recherche, publiés ou non, émanant des établissements d'enseignement et de recherche français ou étrangers, des laboratoires publics ou privés.

$$
\text { Copyright }
$$




\title{
BMP4 patterns Smad activity and generates stereotyped cell fate organization in spinal organoids
}

\author{
Nathalie Duval ${ }^{1,2}$, Célia Vaslin ${ }^{3,4,5, *}$, Tiago C. Barata ${ }^{1, *}$, Youcef Frarma ${ }^{1}$, Vincent Contremoulins ${ }^{1,6}$, \\ Xavier Baudin ${ }^{1,6}$, Stéphane Nedelec ${ }^{3,4,5, \pm}$ and Vanessa C. Ribes ${ }^{1, \pm}$
}

\begin{abstract}
Bone morphogenetic proteins (BMPs) are secreted regulators of cell fate in several developing tissues. In the embryonic spinal cord, they control the emergence of the neural crest, roof plate and distinct subsets of dorsal interneurons. Although a gradient of BMP activity has been proposed to determine cell type identity in vivo, whether this is sufficient for pattern formation in vitro is unclear. Here, we demonstrate that exposure to BMP4 initiates distinct spatial dynamics of BMP signalling within the self-emerging epithelia of both mouse and human pluripotent stem cell-derived spinal organoids. The pattern of BMP signalling results in the stereotyped spatial arrangement of dorsal neural tube cell types, and concentration, timing and duration of BMP4 exposure modulate these patterns. Moreover, differences in the duration of competence time-windows between mouse and human account for the speciesspecific tempo of neural differentiation. Together, this study describes efficient methods for generating patterned subsets of dorsal interneurons in spinal organoids and supports the conclusion that graded BMP activity orchestrates the spatial organization of the dorsal neural tube cellular diversity in mouse and human.
\end{abstract}

KEY WORDS: Bone morphogenetic proteins, Patterning, Organoid, Relay and associating spinal dorsal interneurons, Pluripotent stem cells differentiation, Self-organization

\section{INTRODUCTION}

The rise of methods to generate pluripotent stem cell (PSC)-derived organoids containing multiple cellular subtypes arrayed in threedimensional (3D) structures has opened new avenues to decipher the principles underlying the emergence of patterns of differentiation (Huch et al., 2017; Trujillo and Muotri, 2018). These patterns are likely to be established in response to chemical and mechanical cues applied through culture medium and generated by the endogenous cellular diversity. However, the mechanisms by which organoid cells interpret these cues and acquire specific cell fates largely remain to be determined.

\footnotetext{
${ }^{1}$ Institut Jacques Monod, CNRS UMR7592, Université Paris Diderot, Sorbonne Paris Cité, 75205 Paris Cedex, France. ${ }^{2}$ Institut Pasteur, Department of Developmental and Stem Cell Biology, CNRS URA 2578, 75015 Paris, France. ${ }^{3}$ Inserm, UMR-S 1270, 75005 Paris, France. ${ }^{4}$ Sorbonne Université, Science and Engineering Faculty, 75005 Paris, France. ${ }^{5}$ Institut du Fer à Moulin, 75005 Paris France. ${ }^{6}$ ImagoSeine core facility of Institut Jacques Monod and member of France-Biolmaging.

${ }^{*}$ These authors contributed equally to this work
}

‡Authors for correspondence (vanessa.ribes@ijm.fr; stephane.nedelec@inserm.fr)

D T.C.B., 0000-0002-1483-7990; Y.F., 0000-0002-7996-195X; X.B., 0000-00026002-6347; S.N., 0000-0001-8044-2498; V.C.R., 0000-0001-7016-9192

Received 8 January 2019; Accepted 18 June 2019
To gain insight into these mechanisms, we focused on cellular subtypes generated in the dorsal part of the embryonic spinal cord. The spinal cord originates from the caudal lateral epiblast (CLE) that transits through a pre-neural (PNP) state before acquiring a neurogenic progenitor (NP) state (Henrique et al., 2015). Acquisition of the CLE and PNP states depends on the combined activity of fibroblast growth factor (FGF) and Wnt signalling, while the transition to the NP state is promoted by retinoic acid (RA) signalling. NPs are then directed towards specific neurogenic programmes, depending on their position along the dorso-ventral (D-V) axis of the neural tube (Fig. 1A) (Kalcheim, 2018; Lai et al., 2016). In the dorsal neural tube, NPs comprise six discrete cell types, named from dorsal to ventral as dp1 to dp6 NPs, as well as a group of very dorsal cells that gives rise to neural crest cells (NCCs) and the roof plate (RP) (Fig. 1A). dp4 to dp6 NPs differentiate into five associating interneuron (IN) subtypes: the early born dI4 to dI6 and the late born dILA and dILB. dp 1 to dp3 NPs generate dI 1 to dI3 relay INs. These neurons form functional circuits, in which associating INs transmit the information coming from NCCderived peripheral sensory neurons to relay sensory spinal IN that in turn convey this information to the brain.

The commitment of NPs towards these dorsal fates is determined by specific combinations of transcription factors (TFs) that display stereotypic temporal and spatial expression profiles (Fig. 1A) (Kalcheim, 2018; Lai et al., 2016). The profiles of TFs that mark the NCCs, RP and dp1 to dp3 NPs are, in part, generated in response to gradients of diffusing bone morphogenetic proteins (BMPs) (Kalcheim, 2018; Le Dréau and Martí, 2013; Zagorski et al., 2017). Classical developmental studies led to the model in which the concentration, duration and timing of exposure to BMP are interpreted by PNP and NP cells, guiding them towards a specific fate. Exposing PNP cells explanted from chick embryos to increasing BMP4 concentrations or longer incubations triggers a progressive dorsalization of the generated cell types (Liem et al., 1995; Sasai et al., 2014; Tozer et al., 2013). The emergence of distinct cell fates through modulating BMP receptor activity within chick embryo NPs indicated that in vivo BMP signalling levels may also provide positional information (Timmer et al., 2002; Zechner et al., 2003). Similarly, inhibiting BMP signalling using the antagonist Smad6 at discrete developmental stages reinforced the idea that the duration of BMP signalling discriminates between dorsal cell types (Tozer et al., 2013). Moreover, PNP cells harbour time-regulated competence for dorsal fate specification in response to BMP: the ability of PNP cells to generate NCCs in response to BMP4 being limited in time (Nitzan et al., 2016; Sasai et al., 2014).

However, recent work based on PSC-derived spinal organoids has questioned the morphogenetic potential of BMP (Andrews et al., 2017; Gupta et al., 2018; Meinhardt et al., 2014; Ogura et al., 2018). Exposure to BMP triggered PNP or NP dorsalization in the organoids. However, regardless of the concentration of BMP used, 


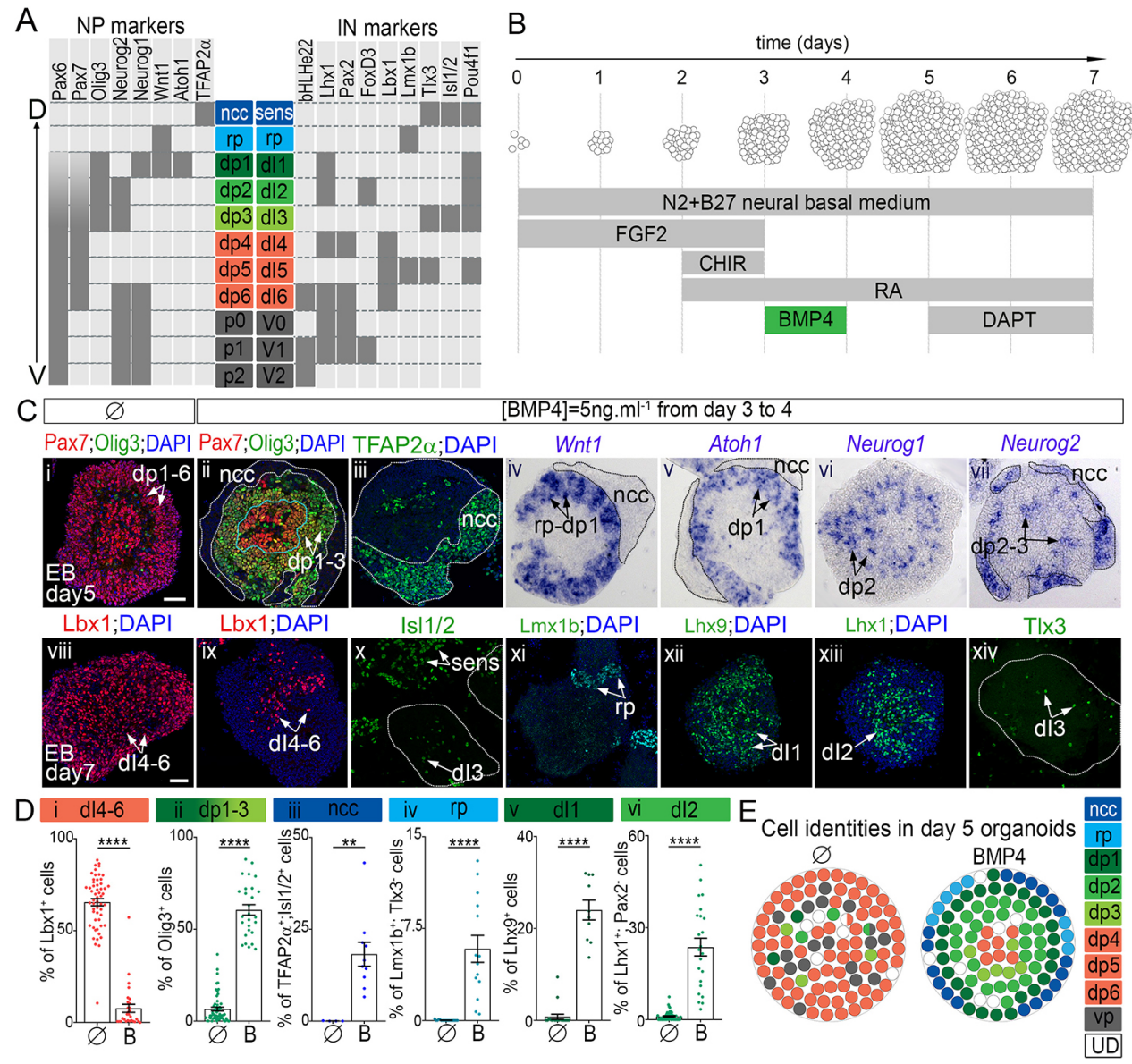

Fig. 1. BMP4 patterns mouse spinal organoids. (A) In vivo D-V expression patterns of neural progenitor (NP) and interneuron (IN) TF markers. (B) Schematics of differentiation conditions that generate dorsal spinal cellular subtypes in EBs.

(C) Immunodetection (i-iii,viii-xiv) and in situ hybridization (iv-vii) for the indicated cell types. White and black outlines in ii-v, vii surround the NCC territory; blue outline in ii indicates cells less responsive to BMP4; white outlines in $x$ and $x i v$ indicate the EB contour. (D) Percentage of cell types harbouring the indicated TF code per image field (individual values; data are mean \pm s.e.m.). ${ }^{* *} P \leq 0.01$ and ${ }^{* * * *} P \leq 0.0001$. (E) Graphs displaying the distribution and percentage of cell subtypes in day 5 organoids; white circles indicate cells with undetermined fate (UD). sens, peripheral sensory neurons. Scale bars: $60 \mu \mathrm{m}$.

several dorsal neural subtypes were generated in a non-organized fashion. This raised the possibility that BMP acts as a permissive, rather than an instructive, signal for the acquisition of dorsal cell fates (Andrews et al., 2017) and that additional signals or mechanisms are responsible for the in vivo spatial organization.

To tackle this issue, we have established robust embryoid body (EB)-based protocols to drive mouse or human PSCs into PNP cells that acquire a dp4 to dp6 state. In these EBs, BMP4 induces concentric patterns of several dorsal neural tube cell types that are reminiscent of those found along the $\mathrm{D}-\mathrm{V}$ axis of the embryo. These patterns depend on the activity of the position-specific temporal profiles of the BMP transcriptional effectors Smad1, Smad5 and Smad9 within the emerging organoid epithelia. Varying the concentration, duration and timing of exposure to BMP4 modifies the cell types generated within these patterns. Furthermore, our data on human organoids revealed that, across evolution, the length of time windows for which PNP and NP cells are competent to generate discrete cell fates in response to BMP4 have been adapted to match species-specific temporal sequences of neural differentiation.

\section{RESULTS AND DISCUSSION}

\section{Efficient generation of associating INs within spinal organoids}

In order to generate dorsal spinal neurons in organoids derived from mouse embryonic stem cells (ESCs), we have adopted 3D EB-based differentiation and adapted culture conditions used for ventral spinal cells generation (Gouti et al., 2014; Maury et al., 2015; Wichterle et al., 2002). EBs were produced in neural basal medium supplemented with FGF2 for the first 3 days of culture and CHIR (CHIR99021), a GSK3 inhibitor, was used to activate Wnt signalling between day 2 and 3 (Fig. 1B). Many day 3 EB cells treated with these two compounds adopted a mesodermal fate, which could be prevented by adding the neuralizing cue RA on day 2 (Fig. 1A,B,D; data not shown) (Beccari et al., 2018; Henrique et al., 2015). CHIR drove the NPs towards caudal $\mathrm{Cdx} 2^{+}$fate (Henrique et al., 2015), while FGF2 further caudalized the state of cells, as shown by the induction of brachial markers such as Hoxc6 (Fig. S1C,D,E) (Liu et al., 2001). In line with in vivo studies (Alvarez-Medina et al., 2008; Lee and Deneen, 2012; Valenta et al., 2011; Zechner et al., 2003), CHIR-mediated Wnt signalling activation favoured dorsal fate acquisition, with $80 \%$ of cells exhibiting a dp4 to dp6 molecular identity (Fig. 1Ci,E, Fig. S2A,Biiii,C). Accordingly, at day 7, following a $48 \mathrm{~h}$-long treatment with Notch signalling inhibitor DAPT (Fig. S3A), EBs contained mainly neurons expressing typical markers of the dI4 to dI6 associating INs (Fig. 1Cviii,Di, Fig. S3B,C). Thus, the identified combination of FGF2, Wnt agonist, RA and DAPT rapidly and efficiently produces mouse ESC-derived organoids containing brachial spinal associating progenitors (day 4, 5) or neurons (day 7) (Andrews et al., 2017; Meinhardt et al., 2014).

\section{Concentric patterns of dorsal neuronal cell types formed in response to BMP4 exposure}

We next examined the effects of BMP4, a BMP ligand implicated in neural patterning (Le Dréau and Martí, 2013), by exposing day 3 NPs to $5 \mathrm{ng} \mathrm{ml}^{-1}$ BMP4 for $24 \mathrm{~h}$ (Fig. 1B). Consistent with BMP4 being a 'dorsalizing' cue, it induced markers of NCCs, RPs, relay dp1/dI1 and dp2/dI2 cells (Fig. 1Cii-vii,ix-xiv,Dii-vi, Figs S2Bivviii"',C,Di, S3B). In day 4 and 5 EBs, these markers displayed 
stereotyped concentric patterns of expression organized along the outer-inner axis of the organoid, with their relative position matching the one found along the $\mathrm{D}-\mathrm{V}$ axis of the neural tube (Fig. 1Cii-vii,E, Fig. S2Bviii). At day 7, the sensory neurons produced from NCCs in the outer ring detached in part from the EB to colonize the dish plate (Fig. 1Cx, not shown). RP cells clustered at the EB periphery and were surrounded by dI1 INs (Fig. 1Cxi, Fig. S3B). Inside the EB, dI2 and a few dI4-6 associating INs were intermingled (Fig. 1Cix, xiii). Altogether, these data demonstrate that, in addition to creating cellular diversity, BMP4 directs spatial arrangement so that the most dorsal cell types are found at a more peripheral position than the ventral ones (Fig. 1E). As shown in the developing spinal cord (Lai et al., 2016), this organization attenuates over time owing to NCC and post-mitotic IN delamination. This may explain why the patterning activity of BMP has not been noticed in previous studies as organoids were analysed solely for terminally differentiated neurons (Andrews et al., 2017; Gupta et al., 2018; Ogura et al., 2018).

\section{Spatial and temporal dynamics in Smad1, Smad5 and Smad 9 activity triggered by BMP4 exposure}

We next sought to investigate whether BMP4-mediated EB patterning could stem from differential spatial and temporal activation of its intracellular downstream effectors. For this, we monitored the levels of the phosphorylated forms of the TFs Smad1, Smad5 and Smad 9 (PSmads) from day 3 to 4 in presence or absence of BMP4 (Fig. 2A-C, Fig. S4).

At all time-points, PSmads ${ }^{+}$cells formed a spatially restricted, three to five cell wide ring, at the periphery of EBs. In contrast, cells deeper in the organoid were devoid of PSmads signal (Fig. 2Avi-x, B, Fig. S4A). This organization could be recapitulated by exposing EB to BMP2 (Fig. S6), but such a spatial restriction in the cell response to signalling cues was not observed for other compounds (not shown) (Maury et al., 2015). This response to BMP was not due to a difference in competence between the outer and inner cells, as single cells obtained from dissociated day 3 EBs homogeneously activated Smads in response to BMP and all cells acquired a NCC state later on (Fig. 2E). This sharp spatial organization could thus emerge from the limited rate of BMP diffusion (Kicheva et al., 2007; Pomreinke et al., 2017; Zinski et al., 2017) or stem from the emergence of a specific tissue organization within EBs. Previous work has demonstrated that the extracellular matrix separating an outer epithelium from inner epithelia can act as a barrier that hampers BMP diffusion (Hu et al., 2004; Ma et al., 2017; Perrimon et al., 2012; Plouhinec et al., 2013; Ramirez and Rifkin, 2009; Wang et al., 2008) (Fig. 2D,F). The emergence of epithelia was revealed by analysing the distribution profiles of Par3, a member of apical protein complexes, and of laminin, a component of the basal lamina (Fig. 2D, Fig. S5i-iv). PSmads ${ }^{+}$cells delineated exactly the outer epithelium whose apical side faced the culture medium (Fig. 2D,F).

The analysis also revealed that P-Smads levels within the outer epithelium varied in space and time. Smads activation displayed a decreasing inward gradient across this epithelium (Fig. 2B, Fig. S4B). The gradient amplitude decreased progressively over time (Fig. 2C), except in the outer-most layer of NCC where, after the initial decline, Smads activity rose again (Fig. 2Ax,C,F, data not shown). The temporal adaptation of BMP signalling was confirmed by the progressive decrease in the levels of BMP4 target genes (Fig. 2Axv, Fig. S2Dii). Negative feedback mediated by the BMP antagonist noggin could be at stake, as its gene was transcriptionally induced $3 \mathrm{~h}$ after BMP4 addition, when PSmads levels had declined (Fig. 2Axi-xv) (Brazil et al., 2015). Progressive depletion of
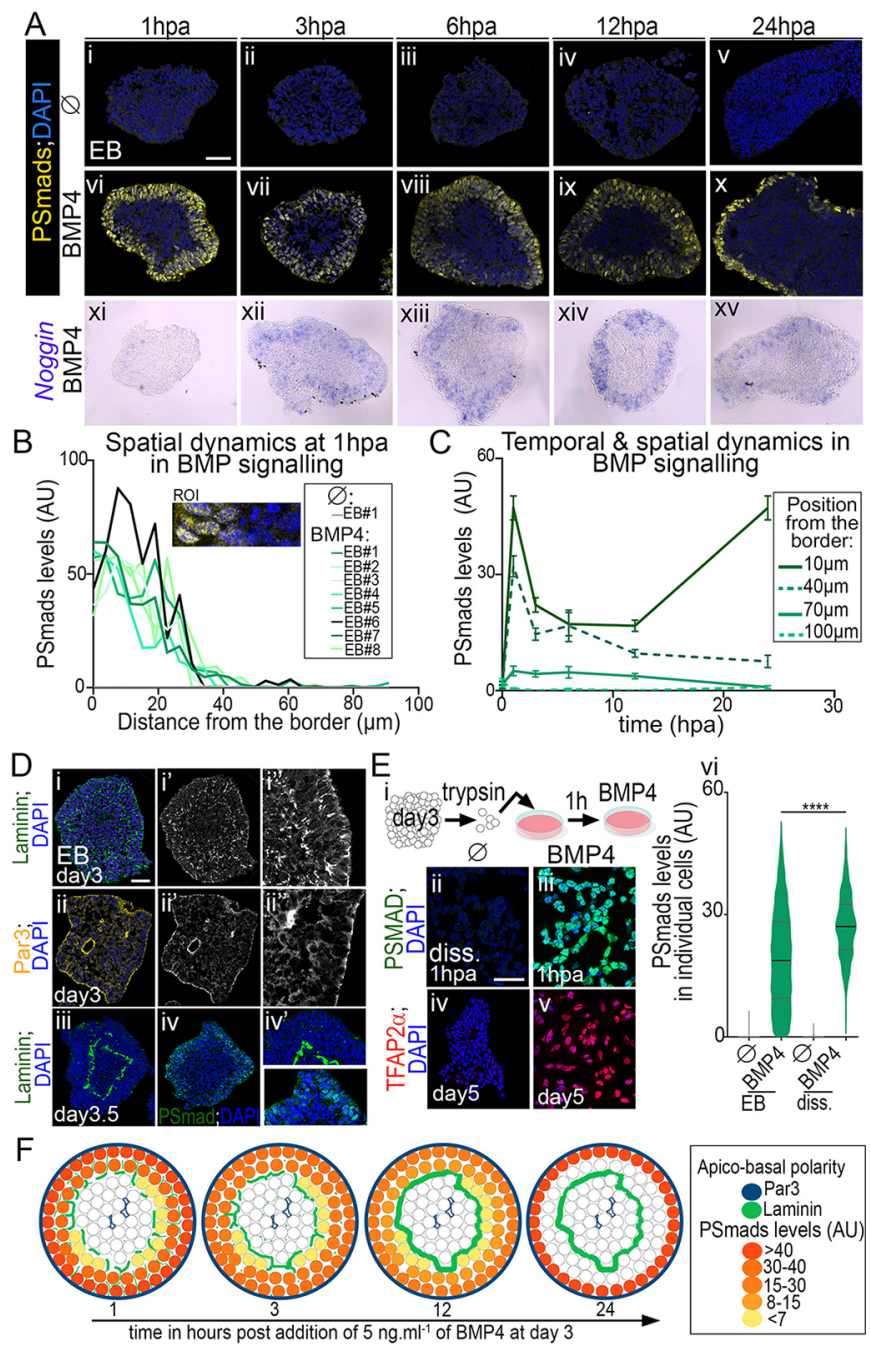

Fig. 2. Spatial and temporal dynamics of PSmads in response to BMP4. (A) Immunodetection of phospho-Smad1, Smad5 and Smad 9 (PSmads), and DAPI labelling ( $\mathrm{i}-\mathrm{x}$ ) and in situ hybridization against Noggin (xi-xv) in EBs cultured without or with $5 \mathrm{ng} \mathrm{ml}^{-1}$ BMP4 (added from day 3) and harvested several hours post-BMP4 addition (hpa). (B) Gradients of PSmads are measured in eight independent regions of interest (ROI; inset) $1 \mathrm{hpa}$.

(C) Temporal and spatial dynamics in PSmad levels in cells at discrete distances from EB borders (data are mean \pm s.e.m.). (D) Laminin, Par3 and PSmad immunodetection, and DAPI labelling in day 3 and 3.5 EBs. (i',ii') Laminin and Par3 staining; ( $\left.\mathrm{i}^{\prime \prime}, \mathrm{ii}^{\prime \prime}\right)$ higher magnification of $\mathrm{i}^{\prime}, \mathrm{ii}^{\prime}$. (E) (i)

Schematics of experimental procedure used to assess the fate of dissociated cells from day 3 EBs exposed to $5 \mathrm{ng} \mathrm{ml}^{-1}$ BMP4 $1 \mathrm{~h}$ post-dissociation. (ii-v) Immunostaining of the indicated markers in dissociated cells $1 \mathrm{hpa}$ (ii,iii) or at day 5 (iv,v). (vi) PSmad levels in EBs or dissociated cells $1 \mathrm{hpa}$ (violin plot; data are mean \pm quartiles). ${ }^{* * *} P \leq 0.0001$. (F) Graphs representing temporal and spatial dynamics in PSmad levels and polarity markers. Scale bars: $60 \mu \mathrm{m}$.

receptors from the cell surface represents an alternative explanation for temporal adaptation (Miller et al., 2019). Together, these data indicate that stereotyped temporal profiles of intracellular signalling are generated in response to BMP4, according to the location of the cell within the organoid (Fig. 2C,F). This spatially graded Smad activity provides an explanation for the inability of BMP4 to drive cells in organoids towards a unique cell type (Andrews et al., 2017; Gupta et al., 2018), which creates an organized cellular diversity. Furthermore, the match between this position-specific signalling dynamics and the emergence of distinct cell types supports a model where 'positional information' results from 
a combination of the levels and the duration of PSmads (Tozer et al., 2013).

\section{Three tuneable morphogenetic parameters of BMP4 exposure discriminate between relay IN subtypes}

To assess directly the morphogenetic potential of BMP4 in organoids, we sought to modulate the three main parameters known to influence cell response to morphogens (Sagner and Briscoe, 2017): the ligand concentration, the exposure duration and the time point at which the ligand is added (Figs S7 and S8).

Increasing BMP4 concentration increased the number of PSmads $^{+}$cells (Fig. S7Avi), the mean levels in PSmads (Fig. S7Avii) as well as the duration for which Smads were active (see insets in Fig. S7Aii-v). Similarly, the duration of BMP4 exposure altered the temporal dynamics of signalling. Nuclear PSmads levels fell $6 \mathrm{~h}$ after BMP4 removal (Fig. S7B), arguing against a long-term memory of intracellular signalling by cells exposed to BMP (Tozer et al., 2013). Importantly, the modulation of PSmad dynamics by increasing BMP4 concentration or duration extended the total amount of BMP dependent cell-types generated and promoted more dorsal cell types at the expense of more ventral ones (Fig. 3A,C,D, Fig. S8A,B).

Changing the time at which EBs were exposed to BMP4 also had strong effects on cell identities (Fig. 3B, Fig. S8C and compare Fig. 1C,D to Fig. 3D). Cells displayed a $12 \mathrm{~h}$ competence time window for generating specific BMP4-dependent cell types, the most dorsal cell types requiring an earliest time of exposure than more ventral ones (Fig. 3Bii-vii). This is in agreement with the limitation in time for chick spinal NP to become NCCs in response to BMP4 (Sasai et al., 2014). Importantly, the switches in cell competence were not due to alteration in the ability of a cell to transduce BMP4 information intracellularly. The amplitude (Fig. S7Cvi), gradient (insets in Fig. S7Cii-iv) and temporal adaptation (not shown) of PSmad profiles remained invariant upon shifts in the timing of BMP4 exposure. Thus, the competence could stem from changes in the molecular state of NPs during the course of their differentiation (Fig. S2Bi-vi) (Sasai et al., 2014).

Finally, in agreement with concentration, duration and timing of exposure being morphogenetic parameters (Sasai et al., 2014; Tozer et al., 2013), we determined conditions needed to generate organoids containing a dominant relay IN subpopulation at their periphery (Fig. 3C,D), including dI2 and dI3, which were poorly generated in previous protocols (Andrews et al., 2017).

\section{Species-specific changes in NP competence time windows are related to the evolution of neuronal differentiation temporality}

Dynamics of neural differentiation and tissue sizes are highly variable between species (Ebisuya and Briscoe, 2018), raising the issue of whether morphogen interpretation is modulated to scale
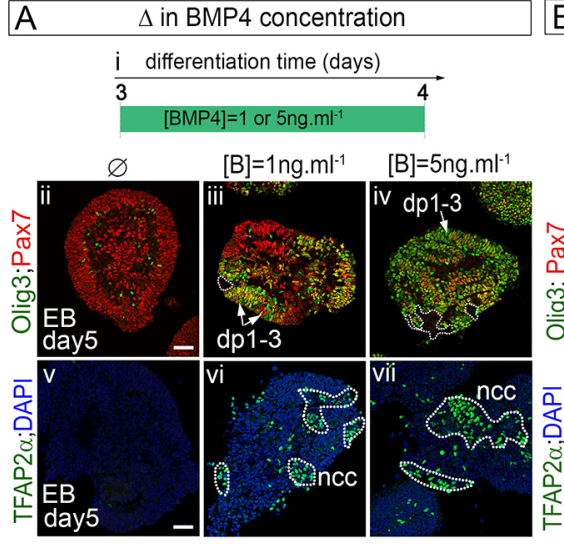

$[\mathrm{B}]=1 \mathrm{ng} \cdot \mathrm{ml}^{-1}$
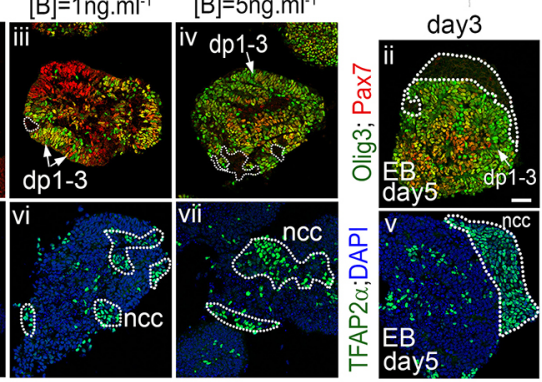

$\Delta$ in [BMP4] and exposure duration from day3.5

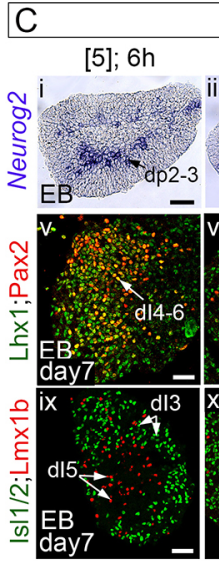

[10]; $6 \mathrm{~h}$
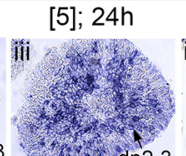

[10]; $24 \mathrm{~h}$
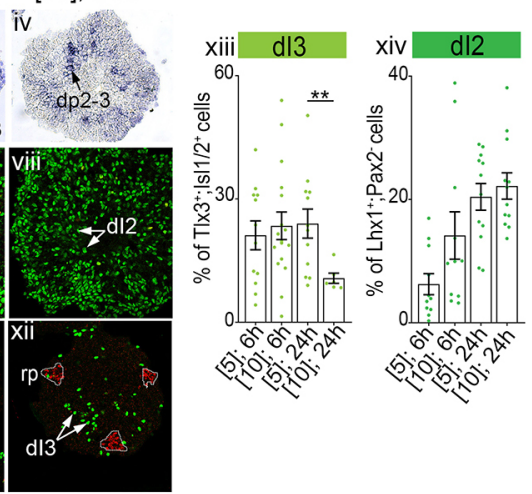

Fig. 3. Morphogenetic parameters of BMP4 exposure generate specific relay IN subtypes. Phenotypic characterization of EBs upon the modulation of BMP4 concentration (A), exposure timing (B) and both BMP4 concentration and exposure duration when adding BMP4 at day 3.5 (C). (Ai,Bi) Schematics indicating BMP4 exposure conditions. (Aii-vii,Bii-vii,Cv-xii) Immunostaining for the indicated markers in EBs grown under the indicated conditions at day 7. (Ci-iv) In situ hybridization for Neurog2 in day 5 EBs cultured under the indicated conditions. (Cxiii,xiv) Percentage of dl2 and dl3 INs harbouring the indicated TF code per image field (individual values; data are mean \pm s.e.m.). ${ }^{* *} P \leq 0.01$. (D) Graphs displaying the distribution and percentage of cell subtypes in day 7 organoids cultured under the indicated conditions. Scale bars: $60 \mu \mathrm{m}$. 
with these dynamics. To address this, we modified our protocol to generate ventral spinal neurons from human iPSCs (Maury et al., 2015) (Fig. 4Ai). As for mouse ESCs, removing the Shh agonist SAG was sufficient to generate spinal organoids containing mostly dp4-dp6 NPs at day 9 of differentiation and associating neurons at day 14 (Fig. 4Aii,iii). Importantly, although both human and mouse dorsal spinal organoids contained similar number of cells (not shown), the time taken to generate these cells in human was around $\sim 2.5$ times longer. Hence, as for motor neuron generation, the speed of differentiation during dorsal differentiation varies greatly between vertebrate species (Maury et al., 2015; Wichterle et al., 2002).

Exposure of hiPSC-derived organoids to BMP4 revealed that the response of human cells is similar to that of mouse cells. Smad activation was restricted to the outer layer of cells, which was separated from more inner cells by a basal lamina (Fig. 4B, Fig. S5v-viii). PSmad levels were higher in the most peripheral cells and were decreased within the width of the epithelium (Fig. 4Bi, ${ }^{\prime}$ ). Accordingly, different cell types were produced in a concentric manner, with the most-dorsal subtypes located at a more peripheral position than the ventral subtypes (Fig. 4D). Finally, both BMP4 concentration and exposure duration influenced the proportion of the BMP-dependent cell types produced (Fig. 4D,E). As in mouse, human NPs displayed competence time windows for the generation of NCCs versus dp1-dp3 cells (Fig. 4C). Yet these windows lasted about twice as long as they did in mouse (compare Fig. 4C with 3B) and paralleled the species-specific time frames of neuronal differentiation. This supports the idea that competence is a consequence of sequential transitions in the transcriptional states of differentiating NPs that occur at slower pace in humans (Sasai et al., 2014).

Together, using efficient and rapid protocols for the differentiation of human and mouse PSC in organoids containing specific subsets of associating and relay spinal neurons, we revealed key parameters for BMP4-mediated tissue patterning. First, 3D polarized epithelia emerging within the organoids participate in the generation of stereotyped profiles of Smad activity and of discrete subsets of cellular subtypes. Their emergence constrains the spatial distribution of not only proteins controlling ligand diffusion, but also of the basolaterally located BMP receptors, which together are likely to generate spatial and temporal dynamics in BMP intracellular signalling (Etoc et al., 2016; Miller et al., 2019; Pomreinke et al., 2017; Wang et al., 2008; Zhang et al., 2018 preprint). Second, as shown for a great number of morphogens (Sagner and Briscoe, 2017), the timing of differentiation, i.e. the molecular state of the receiving cells, appeared to be the most constraining parameter for the response to BMP4, with species specificities. In terms of bioengineering of BMP-dependent cell
A

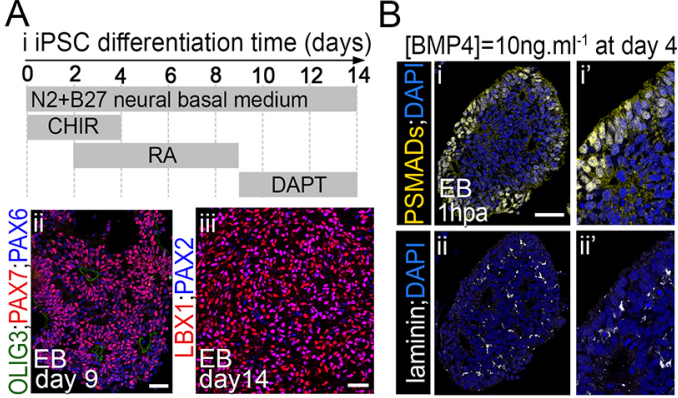

C

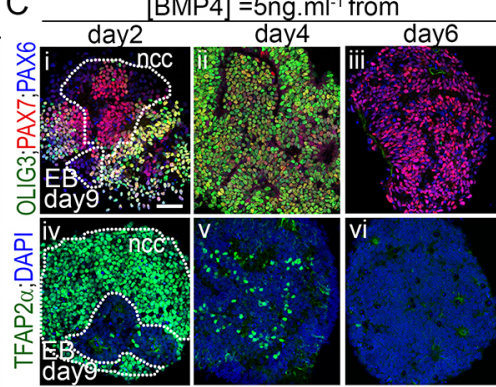

$\mathrm{D}$
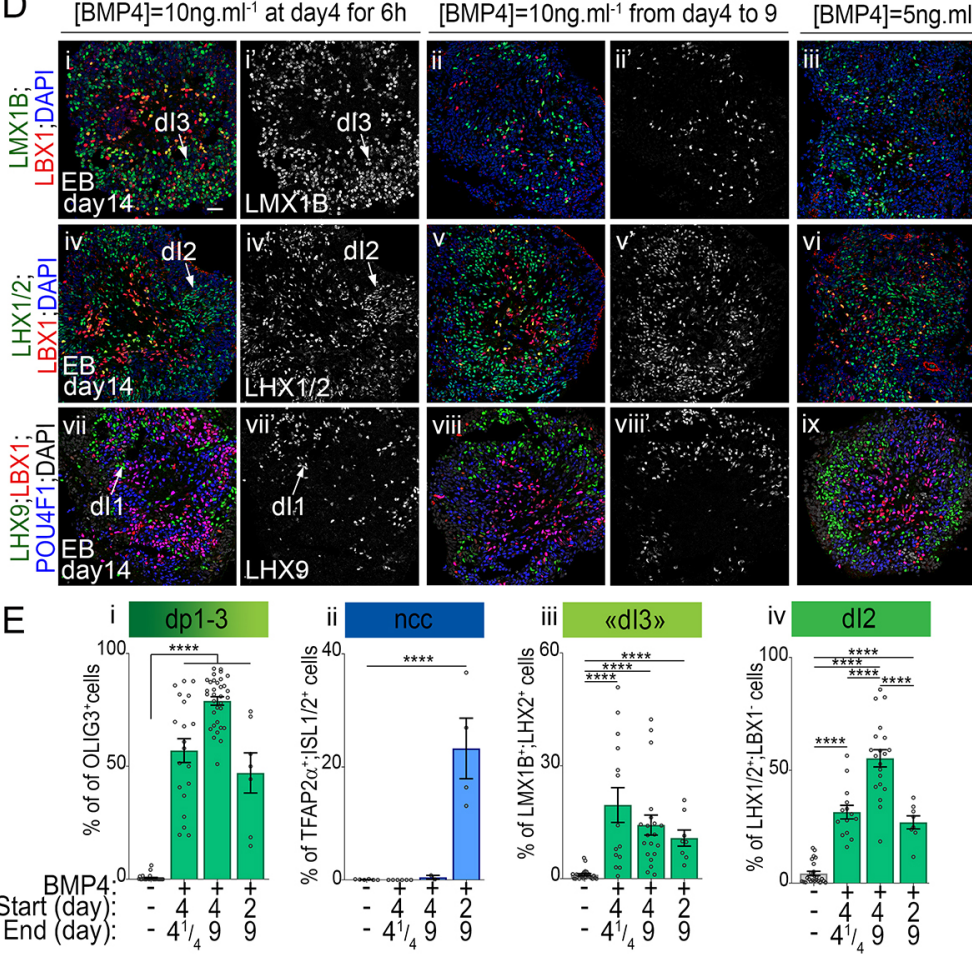
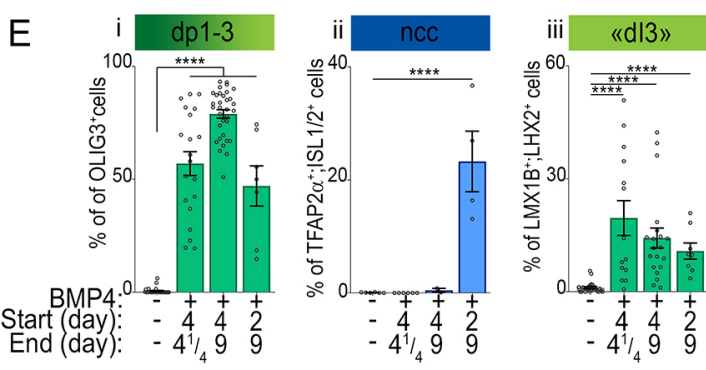
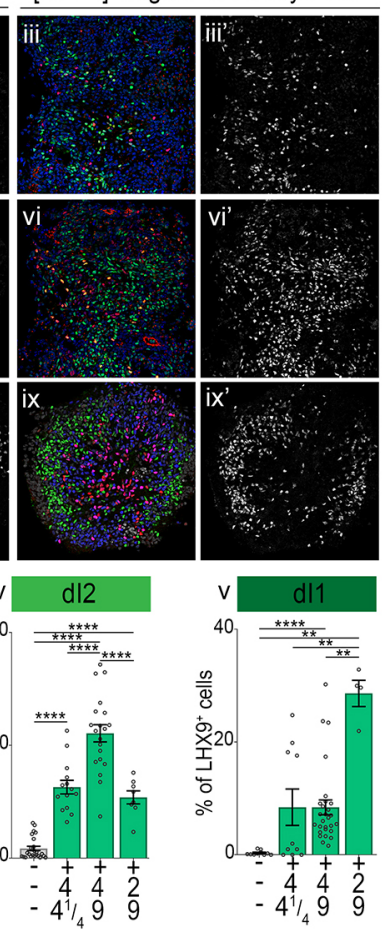

Fig. 4. Morphogenetic effects of BMP4 exposure on human iPSC-derived spinal organoids. (A) Generation of associating progenitors and IN from human iPSCs.

(Ai) Schematics indicating drug treatments to convert human iPSCs into dorsal spinal cells. (Aii,iii) Immunostaining for the indicated NP (ii) and IN (iii) markers in day 9 and 14 organoids.

(B) Immunodetection of pSmads or laminin and DAPI labelling in day 4 organoids treated for $1 \mathrm{~h}$ with BMP4. (C) Immunostaining for the indicated NP markers in day 9 EBs grown in presence of BMP4 added at the indicated time points and maintained up to day 9. (D) Immunostaining for the indicated post-mitotic markers in day $14 \mathrm{EBs}$ grown under the indicated conditions. (E) Percentage of IN subtypes harbouring the indicated identities (individual values; data are mean \pm s.e.m.).

${ }^{* *} P \leq 0.01$ and ${ }^{* * * *} P \leq 0.0001$. Scale bars: $60 \mu \mathrm{m}$. 
types for basic and translational perspectives, our results call for the elaboration of new diffusible BMP pathway activators to generate homogenous populations of cells and for a precise temporal control of morphogen signalling with drug treatments.

\section{MATERIALS AND METHODS}

\section{Cell line maintenance and differentiation}

Mouse ESC line HM1 (Selfridge et al., 1992) at passages ranging from 15 to 19 were maintained on mitotically inactive primary mouse embryo fibroblasts in EmbryoMax D-MEM supplemented with 10\% ESCqualified foetal bovine serum (Millipore), L-glutamine, non-essential amino acid, nucleosides, $0.1 \mathrm{mM} \beta$-mercaptoethanol (Life Technologies) and $1000 \mathrm{U} \mathrm{ml}^{-1}$ leukaemia inhibitory factor (Millipore). Human iPSC WTSIi008-A cell and WTSIi002-A line (EBISC, European Bank for Pluripotent Stem Cells) were cultured in E8 medium on vitronectin (Life Technologies) as previously described (Maury et al., 2015).

To initiate mouse ESC embryoid bodies differentiation, cells were trypsinized and placed twice onto gelatinized tissue culture plates to remove feeders. Cells $\left(5 \times 10^{4}\right.$ cells ml $\left.^{-1}\right)$ were placed in ultra-low attachment petri dishes (Corning) and in Advanced Dulbecco's Modified Eagle/F12 and Neurobasal media (1:1, Life Technologies) supplemented with $1 \times$ B27 devoid of vitamin A and $1 \times \mathrm{N} 2$ (Life Technologies), $2 \mathrm{mM}$ L-glutamine (Life Technologies), $\quad 0.1 \mathrm{mM} \quad \beta$-mercaptoethanol, penicillin and streptomycin (Life Technologies). At this concentration of cells, small EBs were formed from day 1 of differentiation, and grown as such up to day 7 of differentiation; medium was changed every day from day 2 onwards. Human PSC EB differentiation was performed as described by Maury et al. (2015), except the medium did not contain LDN193189 or SB431542. Cells $\left(1.5 \times 10^{5}\right.$ cells $\left.\mathrm{ml}^{-1}\right)$ were seeded in ultra-low attachment six-well plates (Corning). Medium was changed on days 2, 4, 7,9 and 11 of differentiation. Chemical drugs to inhibit or activate key developmental signalling pathways were used at the following concentrations: $10 \mathrm{ng} \mathrm{ml}^{-1} \mathrm{bFGF}$ (FGF2, R\&D), $3 \mu \mathrm{M}$ CHIR99021 (Tocris or Axon Medchem), $10 \mathrm{nM}$ retinoic acid (Sigma), $1 \mathrm{ng} \mathrm{ml}^{-1}$ to $15 \mathrm{ng} \mathrm{ml}^{-1}$ BMP4 (R\&D), $10 \mu \mathrm{M}$ DAPT (Stemgent). Figs 1B and 4A depict the time frames over which these drugs were applied to mouse and human EBs, respectively. Human iPSC experiments were declared (CD-2015-2559) and approved by local ethical Committee (CPP A95)

\section{Dissociation of mouse ESC-derived neuroprogenitors for monolayer culture}

EB at day 3 of differentiation were incubated with $0.05 \%$ trypsin-EDTA for $3 \mathrm{~min}$. After fetal bovine serum (FBS) trypsin inactivation they were dissociated by pipetting and filtered $(40 \mu \mathrm{m}$ pore size). Single cell suspensions were plated at $10^{5}$ cells well $^{-1}$ in a 24 -well plate onto a glass coverslip coated with $20 \mu \mathrm{g} \mathrm{ml}^{-1}$ poly-ornithine (Sigma) and $5 \mu \mathrm{g} \mathrm{ml}^{-1}$ laminin (Life Technologies). Cells were allowed to adhere $1 \mathrm{~h}$ before the addition of fresh differentiation medium containing BMP4.

\section{Expression analyses RT-qPCR}

Total RNA was extracted from 50 to 250 EBs collected at different time points using the NucleoSpin RNA kit (Macherey-Nagel) following manufacturer's instructions. cDNAs were synthetized using SuperScript IV (Thermo Fisher Scientific), random primers and oligo dT. For real-time quantitative PCR (RT-qPCR), SYBR Green I Master (Roche) and the LightCycler 480 II (Roche) were used. PCR primers were designed using Primer3 software (Table S1). Levels of expression per gene for a given time point was measured in biological duplicates or triplicates. Mouse gene expression levels were expressed relatively to TATA-box binding protein (TBP) mRNA levels and normalized to the expression in either GD11.5 dissected spinal cord or mouse ESCs.

\section{Immunofluorescence and in situ hybridization}

EB fixation, embedding and cryosectioning have been described previously (Maury et al., 2015), so have the immunolabelling and in situ hybridization protocols (Briscoe et al., 2000; Yamada et al., 1993). Details of the antibodies are provided in Table S2. Analyses were carried out using a Leica TCS SP5 confocal microscope or a Zeiss Axioplan 2, and images processed with Photoshop 7.0 software (Adobe Systems) or ImageJ v.1.43g image analysis software $(\mathrm{NIH})$.

\section{Quantification}

The number of cells immunolabelled was calculated using Cell Profiler (Broad institute) after nuclei segmentation based on DAPI fluorescence signal with a signal intensity threshold and was expressed as the percentage of all detected nuclei. The EB area labelled by in situ hybridization probes was estimated using ImageJ v.1.43g image analysis software (NIH) and was expressed as the percentage of the whole EB surface. For each condition, these quantifications were performed on a minimum of five images per experiment and on a minimum of two independent experiments. Percentage of all cellular subtypes analysed were represented using a dot plot shaped as a circle; the position of the cellular subtypes reflected our observations. Levels of PSmad fluorescent signal intensity per cells were evaluated using Cell Profiler by subtracting background values from EBs that were not treated with BMP4. Violin plots were used to show the spread of these levels in five images per experiments and on a minimum of three independent experiments. The fluorescence intensity of PSmad expression along the EB outer-inner axis was measured in rectangles $16 \mu \mathrm{m}$ wide and $100 \mu \mathrm{m}$ long positioned perpendicular to an EB tangent line using ImageJ v.1.43g. Background measurements were obtained from EBs not treated with BMP4 and these were subtracted from each assayed profile. Statistical analysis was carried out and graphs were created using Prism Graphpad software. Non-parametric $t$-tests were used to evaluate pair-wise comparisons between conditions. $P$-values are: ${ }^{*} P \leq 0.05,{ }^{*} P \leq 0.01, * * * P \leq 0.001$ and $* * * * P \leq 0.0001$

\section{Whole-mount immunolabelling, 3D scans and image processing}

EBs fixed in 4\% paraformaldehyde and rinsed with PBS were incubated in a blocking solution of $0.5 \%$ Triton X-100 and $1 \%$ BSA for $10 \mathrm{~h}$. They were then incubated for $48 \mathrm{~h}$ with anti-PSmad antibodies (Table S2). After overnight washes in PBS and $0.5 \%$ Triton X-100, they were incubated overnight with a secondary antibody coupled to Alexa A488 (Table S2) After 1 day of washes in PBS and $0.5 \%$ Triton X-100, they were mounted in Vectashield mounting medium (Vector) between coverslips and a glass slide. They were imaged using a Leica TCS SP5 confocal microscope and the image segmentation and signal quantification were processed using Imaris (Bitplane). Notably, surfaces delimitating PSmad-positive nuclei in the EBs were defined using the surface segmentation tool (Fig. S4Ai-i"). Segmentation of PSmad-positive cells was established and the mean intensity values colour coded using the spot detection tool (Fig. S4B). All observations were carried out on at least seven EBs.

\section{Acknowledgements}

We deeply thank C. Birchmeier, T. Müller, J. B. Brunet and A. Pierani for antibodies E. Marti and G. Le Dréau for constructs; and the ImagoSeine core facility of Institut Jacques Monod, a member of France-Biolmaging (ANR-10-INBS-04) and certified IBiSA, as well as the IFM imaging facility. We are grateful to Line Manceau, Benoit Sorre and James Briscoe for critical comments on the manuscript.

\section{Competing interests}

The authors declare no competing or financial interests.

\section{Author contributions}

Conceptualization: N.D., S.N., V.C.R.; Methodology: N.D., C.V., T.C.B., Y.F., X.B., S.N., V.C.R.; Software: V.C.; Validation: N.D., C.V., T.C.B., V.C.R.; Formal analysis: N.D., C.V., T.C.B., Y.F., V.C., V.C.R.; Investigation: N.D., C.V., T.C.B., V.C.R.; Writing - original draft: V.C.R.; Writing - review \& editing: N.D., C.V., S.N., V.C.R.; Visualization: V.C.; Supervision: S.N., V.C.R.; Funding acquisition: S.N., V.C.R.

\section{Funding}

S.N. and V.C.R. are Institut National de la Santé et de la Recherche Médicale researchers; N.D. is employed by the Institut Pasteur. Work in the lab of V.R. is supported by a Centre National pour la Recherche Scientifique/Institut National de la Santé et de la Recherche Médicale ATIP-AVENIR program, as well as by a Ligue Nationale Contre le Cancer grant (PREAC2016.LCC). Studies in the lab of S.N. are funded by an Institut National de la Santé et de la Recherche Médicale ATIP- 
AVENIR program co-sponsored by the Association Française contre les Myopathies (AFM-telethon) and a chair of excellence of the Laboratoire d'Excellence de Biologie pour la Psychiatrie (Bio-Psy) (11-LABX-0035).

\section{Supplementary information}

Supplementary information available online at

http://dev.biologists.org/lookup/doi/10.1242/dev.175430.supplemental

\section{References}

Alvarez-Medina, R., Cayuso, J., Okubo, T., Takada, S. and Marti, E. (2008). Wnt canonical pathway restricts graded Shh/Gli patterning activity through the regulation of Gli3 expression. Development 135, 237-247. doi:10.1242/dev. 012054

Andrews, M. G., del Castillo, L. M., Ochoa-bolton, E., Yamauchi, K., Smogorzewski, J., Butler, S. J., Castillo, L. M., Ochoa-bolton, E., Yamauchi, K., Smogorzewski, J. et al. (2017). BMPs direct sensory interneuron identity in the developing spinal cord using signal-specific not morphogenic activities. eLife 6, e30647. doi:10.7554/eLife.30647

Beccari, L., Moris, N., Girgin, M., Turner, D. A., Baillie-Johnson, P., Cossy, A.C., Lutolf, M. P., Duboule, D. and Arias, A. M. (2018). Multi-axial selforganization properties of mouse embryonic stem cells into gastruloids. Nature 562, 272-276. doi:10.1038/s41586-018-0578-0

Brazil, D. P., Church, R. H., Surae, S., Godson, C. and Martin, F. (2015). BMP signalling: agony and antagony in the family. Trends Cell Biol. 25, 249-264. doi:10. 1016/j.tcb.2014.12.004

Briscoe, J., Pierani, A., Jessell, T. M. and Ericson, J. (2000). A homeodomain protein code specifies progenitor cell identity and neuronal fate in the ventral neural tube. Cell 101, 435-445. doi:10.1016/S0092-8674(00)80853-3

Ebisuya, M. and Briscoe, J. (2018). What does time mean in development? Development 145, dev164368. doi:10.1242/dev.164368

Etoc, F., Metzger, J., Ruzo, A., Kirst, C., Yoney, A., Ozair, M. Z., Brivanlou, A. H. and Siggia, E. D. (2016). A balance between secreted inhibitors and edge sensing controls gastruloid self-organization. Dev. Cell 39, 302-315. doi:10.1016/ j.devcel.2016.09.016

Gouti, M., Tsakiridis, A., Wymeersch, F. J., Huang, Y., Kleinjung, J., Wilson, V. and Briscoe, J. (2014). In vitro generation of neuromesodermal progenitors reveals distinct roles for wnt signalling in the specification of spinal cord and paraxial mesoderm identity. PLoS Biol. 12, e1001937. doi:10.1371/journal.pbio. 1001937

Gupta, S., Sivalingam, D., Hain, S., Makkar, C., Sosa, E., Clark, A. and Butler, S. J. (2018). Deriving dorsal spinal sensory interneurons from human pluripotent stem cells. Stem Cell Rep. 10, 390-405. doi:10.1016/j.stemcr.2017.12.012

Henrique, D., Abranches, E., Verrier, L. and Storey, K. G. (2015). Neuromesodermal progenitors and the making of the spinal cord. Development 142, 2864-2875. doi:10.1242/dev.119768

Hu, Q., Ueno, N. and Behringer, R. R. (2004). Restriction of BMP4 activity domains in the developing neural tube of the mouse embryo. EMBO Rep. 5, 734-739. doi:10.1038/sj.embor.7400184

Huch, M., Knoblich, J. A., Lutolf, M. P. and Martinez-arias, A. (2017). The hope and the hype of organoid research. Development 144, 938-941. doi:10.1242/dev. 150201

Kalcheim, C. (2018). Neural crest emigration: from start to stop. Genesis 56 e23090. doi:10.1002/dvg.23090

Kicheva, A., Pantazis, P., Bollenbach, T., Kalaidzidis, Y., Bittig, T., Julicher, F. and Gonzalez-Gaitan, M. (2007). Kinetics of morphogen gradient formation Science 315, 521-525. doi:10.1126/science.1135774

Lai, H. C., Seal, R. P. and Johnson, J. E. (2016). Making sense out of spinal cord somatosensory development. Development 143, 3434-3448. doi:10.1242/dev. 139592

Le Dréau, G. and Martí, E. (2013). The multiple activities of BMPs during spinal cord development. Cell. Mol. Life Sci. 70, 4293-4305. doi:10.1007/s00018-013-1354-9

Lee, H. K. and Deneen, B. (2012). Daam2 is required for dorsal patterning via modulation of canonical Wnt signaling in the developing spinal cord. Dev. Cell 22 183-196. doi:10.1016/j.devcel.2011.10.025

Liem, K. F., Tremml, G., Roelink, H. and Jessell, T. M. (1995). Dorsal differentiation of neural plate cells induced by BMP-mediated signals from epidermal ectoderm. Cell 82, 969-979. doi:10.1016/0092-8674(95)90276-7

Liu, J.-P., Laufer, E. and Jessell, T. M. (2001). Assigning the positional identity of spinal motor neurons: rostrocaudal patterning of Hox-c expression by FGFs, Gdf11, and retinoids. Neuron 32, 997-1012. doi:10.1016/S0896-6273(01)00544-X

Ma, M., Cao, X., Dai, J. and Pastor-Pareja, J. C. (2017). Basement membrane manipulation in Drosophila wing discs affects Dpp retention but not growth mechanoregulation. Dev. Cell 42, 97-106.e4. doi:10.1016/j.devcel.2017.06.004

Maury, Y., Côme, J., Piskorowski, R. A., Salah-Mohellibi, N., Chevaleyre, V., Peschanski, M., Martinat, C. and Nedelec, S. (2015). Combinatorial analysis of developmental cues efficiently converts human pluripotent stem cells into multiple neuronal subtypes. Nat. Biotechnol. 33, 89-96. doi:10.1038/nbt.3049
Meinhardt, A., Eberle, D., Tazaki, A., Ranga, A., Niesche, M., WilschBräuninger, M., Stec, A., Schackert, G., Lutolf, M. and Tanaka, E. M. (2014) 3D reconstitution of the patterned neural tube from embryonic stem cells. Stem Cell Rep. 3, 987-999. doi:10.1016/j.stemcr.2014.09.020

Miller, D. S. J., Schmierer, B. and Hill, C. S. (2019). TGF- $\beta$ family ligands exhibit distinct signaling dynamics that are driven by receptor localization. J. Cell Sci. 146, jcs.234039. doi:10.1242/jcs.234039

Nitzan, E., Avraham, O., Kahane, N., Ofek, S., Kumar, D. and Kalcheim, C. (2016). Dynamics of BMP and Hes1/Hairy1 signaling in the dorsal neural tube underlies the transition from neural crest to definitive roof plate. BMC Biol. 14, 23 . doi:10.1186/s12915-016-0245-6

Ogura, T., Sakaguchi, H., Miyamoto, S. and Takahashi, J. (2018). Threedimensional induction of dorsal, intermediate and ventral spinal cord tissues from human pluripotent stem cells. Development 145, dev162214. doi:10.1242/dev. 162214

Perrimon, N., Pitsouli, C. and Shilo, B.-Z. (2012). Signaling mechanisms controlling cell fate and embryonic patterning. Cold Spring Harb. Perspect. Biol. 4, a005975. doi:10.1101/cshperspect.a005975

Plouhinec, J.-L., Zakin, L., Moriyama, Y. and De Robertis, E. M. (2013). Chordin forms a self-organizing morphogen gradient in the extracellular space between ectoderm and mesoderm in the Xenopus embryo. Proc. Natl. Acad. Sci. USA 110 20372-20379. doi:10.1073/pnas.1319745110

Pomreinke, A. P., Soh, G. H., Rogers, K. W., Bergmann, J. K., Bläßle, A. J. and Müller, P. (2017). Dynamics of BMP signaling and distribution during zebrafish dorsal-ventral patterning. eLife 6, e25861. doi:10.7554/eLife.25861

Ramirez, F. and Rifkin, D. B. (2009). Extracellular microfibrils: contextual platform for TGF $\beta$ and BMP signaling. Curr. Opin. Cell Biol. 21, 616-622. doi:10.1016/j.ceb. 2009.05.005

Sagner, A. and Briscoe, J. (2017). Morphogen interpretation: concentration, time, competence, and signaling dynamics. Wiley Interdiscip. Rev. Dev. Biol. 6, e271. doi:10.1002/wdev.271

Sasai, N., Kutejova, E. and Briscoe, J. (2014). Integration of signals along orthogonal axes of the vertebrate neural tube controls progenitor competence and increases cell diversity. PLoS Biol. 12, e1001907. doi:10.1371/journal.pbio. 1001907

Selfridge, J., Pow, A. M., McWhir, J., Magin, T. M. and Melton, D. W. (1992). Gene targeting using a mouse HPRT minigene/HPRT-deficient embryonic stem cell system: inactivation of the mouse ERCC-1 gene. Somat. Cell Mol. Genet. 18 325-336. doi:10.1007/BF01235756

Timmer, J. R., Wang, C. and Niswander, L. (2002). BMP signaling patterns the dorsal and intermediate neural tube via regulation of homeobox and helix-loophelix transcription factors. Development 129, 2459-2472.

Tozer, S., Le Dréau, G., Marti, E., Briscoe, J. and Le Dreau, G. (2013). Tempora control of BMP signalling determines neuronal subtype identity in the dorsal neural tube. Development 140, 1467-1474. doi:10.1242/dev.090118

Trujillo, C. A. and Muotri, A. R. (2018). Brain organoids and the study of neurodevelopment. Trends Mol. Med. 24, 982-990. doi:10.1016/j.molmed.2018. 09.005

Valenta, T., Gay, M., Steiner, S., Draganova, K., Zemke, M., Hoffmans, R. Cinelli, P., Aguet, M., Sommer, L. and Basler, K. (2011). Probing transcriptionspecific outputs of beta-catenin in vivo. Genes Dev. 25, 2631-2643. doi:10.1101/ gad. 181289.111

Wang, X., Harris, R. E., Bayston, L. J. and Ashe, H. L. (2008). Type IV collagens regulate BMP signalling in Drosophila. Nature 455, 72-77. doi:10.1038/ nature 07214

Wichterle, H., Lieberam, I., Porter, J. A. and Jessell, T. M. (2002). Directed differentiation of embryonic stem cells into motor neurons. Cell 110, 385-397. doi:10.1016/S0092-8674(02)00835-8

Yamada, T., Pfaff, S. L., Edlund, T. and Jessell, T. M. (1993). Control of cell pattern in the neural tube: motor neuron induction by diffusible factors from notochord and floor plate. Cell 73, 673-686. doi:10.1016/0092-8674(93)90248-O

Zagorski, M., Tabata, Y., Brandenberg, N., Lutolf, M. P., Tkačik, G., Bollenbach, T., Briscoe, J. and Kicheva, A. (2017). Decoding of position in the developing neural tube from antiparallel morphogen gradients. Science 356, 1379-1383. doi:10.1126/science.aam5887

Zechner, D., Fujita, Y., Hülsken, J., Müller, T., Walther, I., Taketo, M. M., Crenshaw, E. B., Birchmeier, W. and Birchmeier, C. (2003). $\beta$-Catenin signals regulate cell growth and the balance between progenitor cell expansion and differentiation in the nervous system. Dev. Biol. 258, 406-418. doi:10.1016/ S0012-1606(03)00123-4

Zhang, Z., Zwick, S., Loew, E., Grimley, J. S. and Ramanathan, S. (2018). Embryo geometry drives formation of robust signaling gradients through receptor localization. bioRxiv, 491290. doi:10.1101/491290

Zinski, J., Bu, Y., Wang, X., Dou, W., Umulis, D. and Mullins, M. C. (2017) Systems biology derived source-sink mechanism of bmp gradient formation. eLife 6, e22199. doi:10.7554/eLife.22199 